\title{
CRESCIMENTO, ACÚMULO DE FÓSFORO E FRAÇÕES FOSFATADAS EM MUDAS DE SETE ESPÉCIES ARBÓREAS NATIVAS ${ }^{1}$
}

\author{
José Zilton Lopes Santos², Álvaro Vilela de Resende ${ }^{3}$, Antônio Eduardo Furtini Neto ${ }^{4}$ e Edmar Fernando Corte ${ }^{5}$
}

\begin{abstract}
RESUMO - Este trabalho objetivou comparar o crescimento, a absorção de fósforo (P) e a distribuição das frações fosfatadas em mudas de sete espécies florestais classificadas como: pioneiras [aroeira (Lithraea molleoides), aroeirinha (Shcinus terebinthifolius), sabiá (Mimosa caesalpiniaefolia), sesbânia (Sesbania virgata)] ou clímax [jatobá (Hymenaea courbaril), guanandi (Calophyllum brasiliensis) e óleo-bálsamo (Myroxylon peruiferum)]. As plantas foram cultivadas com cinco doses de $\mathrm{P}\left(0,100,250,500\right.$ e $\left.800 \mathrm{mg} \mathrm{dm}^{-3} \mathrm{de} P\right)$ em casa de vegetação. Aos 90 dias, as plantas foram colhidas, determinando-se a produção de matéria seca e o conteúdo de $\mathrm{P}$ da parte aérea e as frações de $\mathrm{P}$ total, $\mathrm{P}$ inorgânico e P orgânico presentes nas folhas. As espécies apresentaram grande variação no comportamento diante da adubação fosfatada. De modo geral, as espécies pioneiras foram mais eficientes em produzir matéria seca da parte aérea e na absorção de $\mathrm{P}$ do que as climácicas. Entre as espécies pioneiras, a aroeira apresentou produção de massa estreitamente relacionada ao acúmulo de $\mathrm{P}$, e as proporções de fósforo inorgânico e orgânico permanecem inalteradas com o aumento da disponibilidade do nutriente. O crescimento de aroeirinha, sesbânia e jatobá não acompanhou a absorção do nutriente que é acumulado na forma de fósforo inorgânico na primeira e fósforo orgânico nas últimas. As espécies clímácicas guanandi e óleo-bálsamo apresentaram maior presença de fósforo orgânico, porém a absorção do nutriente e o crescimento das plantas sofreram pouca interferência da adubação fosfatada.
\end{abstract}

Palavras-chave: Espécies florestais, adubação fosfatada e formas de fósforo.

\section{GROWTH, PHOSPHORUS ACCUMULATION AND P FRACTIONS IN SEEDLINGS OF SEVEN NATIVE TREE SPECIES}

\begin{abstract}
This work aimed to compare growth, phosphorus $(P)$ uptake and distribution of P-fractions in seedlings of seven tree species classified as pioneers (Lithraea molleoides, Shcinus terebinthifolius, Mimosa caesalpiniaefolia, Sesbania virgata) or climaxes (Hymenaea courbaril, Calophyllum brasiliensis, Myroxylon peruiferum $)$ and cultivated under five doses of $P\left(0,100,250,500\right.$ and $800 \mathrm{mg} \mathrm{dm}^{-3}$ of $\left.P\right)$ in a greenhouse. $U p$ to ninety days after germination, the plants were harvested, and dry matter, $P$-content and $P$-fractions (total-P, inorganic-P and organic- $P$ ) in the leaves were determined. The tree species presented a wide behavior variation regarding $P$-fertilization, with the pioneer species being more efficient in producing dry matter and absorbing $P$. The biomass production of the pioneer species Lithraea molleoides was directly related to $P$ accumulation. Its proportions of inorganic- $P$ and organic- $P$ remained unaffected by the increased availability of $P$. The growth of the Shcinus terebinthifolius, Sesbania virgata and Hymenaea courbaril did not keep up with $P$ uptake, which was mainly accumulated as inorganic-P in the first species and as organic-P in the two last ones. Climax species Calophyllum brasiliensis and Myroxylon peruiferum presented a larger proportion of organic-P, however, the P uptake and plant growth were little affected by P-fertilization.
\end{abstract}

Keywords: Tree species, P-fertilization and phosphorus forms.

\footnotetext{
${ }^{1}$ Recebido em 24.08.2007 e aceito para publicação em 22.08.2008.

${ }^{2}$ Programa de Pós-Graduação em Ciência do Solo da Universidade Federal de Lavras (UFLA). E-mail: <ziltton@ yahoo.com.br>.

${ }^{3}$ Centro de Pesquisa Agropecuária dos Cerrados da EMBRAPA, Brasília - DF. E-mail: E-mail: <alvaro@cpac.embrapa.br>.

${ }^{4}$ Departamento de Ciência do Solo da Universidade Federal de Lavras (UFLA). E-mail: <afurtini@ufla.br>.

${ }^{5}$ Programa de Iniciação Científica do Departamento de Ciência do Solo da UFLA. E-mail: <afurtini@ufla.br>.
} 


\section{INTRODUÇÃO}

A disponibilidade de nutrientes está entre os fatores que condicionam o desenvolvimento, proliferação e abundância das espécies florestais. Entre os nutrientes, o fósforo (P) é um dos que tem merecido maior preocupação, em razão da sua baixa disponibilidade natural em solos mais intemperizados. São escassas as informações sobre as exigências nutricionais de espécies florestais, em especial das espécies brasileiras (SHUMACHER et al., 2004). A grande diversidade de espécies florestais nativas, com comportamento nutricional distinto (SIQUEIRA et al., 1995), torna a obtenção de informações sobre demanda nutricional e resposta à fertilização de fundamental importância para o êxito dos programas de reflorestamento.

De modo geral, as espécies pioneiras têm seu potencial de crescimento restringido quando se desenvolvem em solos pobres, mostrando-se bastante responsivas à fertilização, ao passo que com o avanço do grupo sucessional o estímulo ao crescimento proporcionado pela adubação é menos pronunciado e, algumas vezes, inexistente; tendência, em parte, atribuída ao crescimento mais lento, característico das espécies clímácicas. Conforme Marschner (1991) e Lambers e Poorter (1992), espécies de crescimento lento apresentam baixa resposta ao fornecimento de nutrientes, característica, em alguns casos, relacionada à adaptação a solos pouco férteis.

A demanda de $\mathrm{P}$ pelas espécies está associada a diversos fatores, como tamanho e conteúdo de $\mathrm{P}$ das sementes, grau de desenvolvimento do sistema radicular, dependência micorrízica, taxa de crescimento e estádio de desenvolvimento da planta. Maior resposta ao fornecimento de $\mathrm{P}$ é esperada em espécies de sementes pequenas e com baixos conteúdos de $\mathrm{P}$, com sistema radicular pouco desenvolvido, com maior capacidade micotrófica e com maior taxa de crescimento e na fase inicial de desenvolvimento. Dessa forma, é de se esperar que as espécies pioneiras sejam mais responsivas às adubações fosfatadas, quando comparadas com as espécies clímácicas (FURTININETOetal., 2000; RESENDE et al., 2005).

Quando submetidos a mesma condição de fertilidade do solo, é comum encontrar comportamentos contrastantes entre espécies e entre genótipos, quanto à nutrição e crescimento. Furtini Neto et al. (1996), avaliando a eficiência nutricional de mudas de Eucalyptus em função de doses de $\mathrm{P}$, observaram que as espécies responderam, de maneira distinta, à aplicação do nutriente. Geralmente, quando uma planta se encontra numa situação de baixa disponibilidade de $\mathrm{P}$, ela tende a utilizá-lo de forma mais eficiente, contrariamente ao que se observa quando a planta é submetida a uma condição de elevada disponibilidade (BALIEIRO et al., 2001).

A resposta distinta das espécies vegetais à fertilização fosfatada tem sido explicada pela dinâmica das frações de P na planta. A avaliação dos teores de $\mathrm{P}$ inorgânico ( $\mathrm{Pi})$ e de $\mathrm{P}$ orgânico $(\mathrm{Po})$ contribui para o entendimento quanto ao crescimento e uso do nutriente pelas plantas (FABRES et al., 1987; FURTINI NETO, 1994; FERNANDES et al., 2000a). Acredita-se que o tamanho do estoque não-metabólico e a habilidade da planta em translocar esse estoque de um compartimento para outro certamente afeta a adaptabilidade e sobrevivência sob condições de estresse nutricional (MARTINEZ et al., 2005). O objetivo deste trabalho foi comparar a resposta em crescimento e absorção de $\mathrm{P}$ e a distribuição das frações fosfatadas em mudas de sete espécies florestais submetidas à adubação com P.

\section{MATERIAL E MÉTODOS}

O experimento foi conduzido em casa de vegetação do Departamento de Ciência do Solo da Universidade Federal de Lavras, em Lavras, MG. Utilizou-se amostra superficial $(0-20 \mathrm{~cm})$ de um Latossolo Vermelho distrófico, argiloso, classificado conforme a Embrapa (1999). Antes da instalação do experimento, o solo foi analisado, e os atributos químicos e físicos são apresentados na Tabela 1. O delineamento experimental adotado foi o inteiramente casualizado, com cinco repetições, num fatorial completo 7 x 5 (sete espécies e cinco doses de P). As espécies estudadas foram: aroeira (Lithraea molleoides Vell. Engl.), aroeirinha (Shcinus terebinthifolius Raddi), sabiá (Mimosa caesalpiniaefolia Benth.), sesbânia (Sesbania virgata Merril.), jatobá (Hymenaea courbaril L.), guanandi (Calophyllum brasiliensis Camb.) e óleo-bálsamo (Myroxylon peruiferum L. f.). As quatro primeiras são classificadas como espécies pioneiras e as demais, como clímax (DAVIDE et al., 1995). O fornecimento de $\mathrm{P}$ correspondeu à aplicação de $0,100,250,500$ e $800 \mathrm{mg} \mathrm{dm}^{-3}$ do nutriente. Detalhes sobre a composição dos tratamentos foram descritos por Resende (1997). 
Tabela 1 - Principais atributos químicos e físicos do Latossolo Vermelho utilizado no experimento Table 1 - Main chemical and physical characteristics of Red Latosol used in the experiment

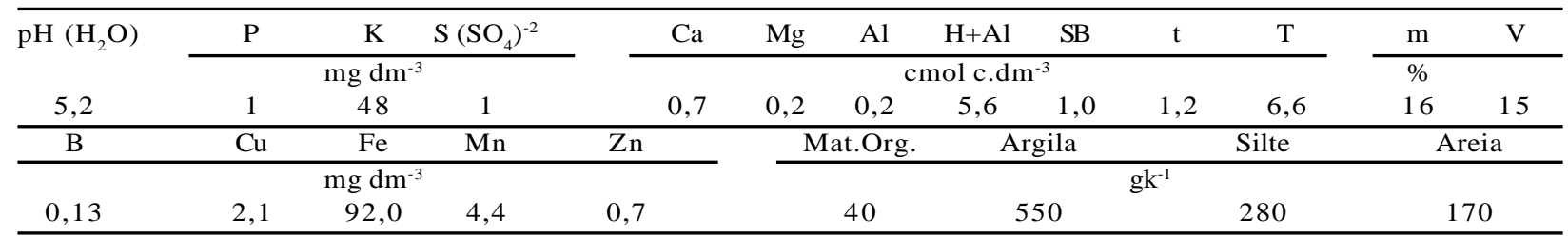

As sementes foram postas para germinar em vermiculita expandida, em datas diferenciadas conforme o período necessário à germinação de cada espécie, proporcionando a obtenção das mudas numa mesma época. Após a emergência das plântulas, procedeuse ao transplantio de todas as mudas ao mesmo tempo para o solo com os tratamentos, conduzindo-se duas plantas por vaso.

Antes da aplicação dos tratamentos, foi realizada a correção da acidez do solo para atingir valor de $\mathrm{pH}$ próximo de 6,0. Em todas as parcelas, efetuou-se uma adubação básica com $100 ; 100 ; 45 ; 0,5 ; 1,5 ; 3,6 ; 0,15$; e 5,0 $\mathrm{mg} \mathrm{dm}^{-3}$ de $\mathrm{N}, \mathrm{K}, \mathrm{S}, \mathrm{B}, \mathrm{Cu}, \mathrm{Mn}, \mathrm{Mo}$ e $\mathrm{Zn}$, respectivamente. $\mathrm{O}$ fornecimento de $\mathrm{P}$ e a fertilização básica foram efetuados com o uso de sais p.a.e balanceados para que somente a quantidade de $\mathrm{P}$ variasse. Os tratamentos e as fertilizações foram realizados individualmente, em cada vaso, com capacidade para três $\mathrm{dm}^{3}$ de substrato. Precedendo o transplantio, o solo com corretivo e fertilizantes foi incubado durante 20 dias. Durante o período experimental, a umidade do solo foi mantida em torno de $60 \%$ do VTP (FREIRE et al., 1980), por meio de irrigações diárias com água deionizada. Três adubações complementares com $\mathrm{N}$ em cobertura, na forma de $\mathrm{NH}_{4} \mathrm{NO}_{3}$, foram efetuadas no decorrer do experimento, totalizando um fornecimento suplementar de 45 e $70 \mathrm{mg} \mathrm{dm}^{-3} \mathrm{de} \mathrm{N}$, na dose $0 \mathrm{de}$ $\mathrm{P}$ e nas demais doses, respectivamente.

Aos 90 dias, as plantas foram colhidas e o material vegetal posto a secar em estufa de circulação forçada de ar a $60^{\circ} \mathrm{C}$ até peso constante, para determinação da matéria seca da parte aérea das espécies nos diversos tratamentos. O material da parte aérea foi moído e submetido à digestão nítrico-perclórica para determinação dos teores de P-total (MALAVOLTA et al., 1997). Com base nos teores de $\mathrm{P}$ e na produção de matéria seca, foi calculado o acúmulo do nutriente. Utilizando-se os resultados obtidos na dose zero de $\mathrm{P}$ como referência, foram calculados os incrementos porcentuais na produção de matéria seca (MS\%) e no conteúdo de fósforo (CP\%) das plantas nas demais doses.

No momento do corte das plantas, foi colhida a primeira folha fisiologicamente madura (completamente formada) do ápice para a base, sendo as amostras colocadas em ácido perclórico $0,2 \mathrm{~N}$ e congeladas. Nessas amostras, foram determinados o P-total solúvel em ácido (Pts), o P-inorgânico solúvel em ácido $(\mathrm{Pi})$ e, por diferença, o P-orgânico solúvel em ácido (Po), conforme método de Smilie e Krotkov (1960) e Hogue et al. (1970), modificado por Martinez (1992).

Modelos de regressão foram ajustados para produção de matéria seca da parte aérea (MSPA), conteúdo de P na parte aérea (CPPA), incrementos porcentuais de matéria seca (MS\%) e de conteúdo de $\mathrm{P}(\mathrm{CP} \%)$ na parte aérea, teor de Pts e participação porcentual das frações de fósforo (Pi e Po) na folha, como variáveis dependentes das doses de $\mathrm{P}$ para cada espécie, utilizando-se o programa estatístico SISVAR 4.6 (FERREIRA, 2000).

\section{RESULTADOS E DISCUSSÃO}

\subsection{Crescimento das plantas e absorção de $P$}

De modo geral, ao elevar o fornecimento de $\mathrm{P}$ ocorreram aumentos na produção de matéria seca da parte aérea (MS) das espécies pioneiras: aroeira, aroeirinha e sesbânia (Figuras 1A, 2A e 3A). Os ganhos significativos em crescimento evidenciam a elevada responsividade dessas espécies à adubação fosfatada. Aumentos no crescimento inicial de espécies arbóreas pioneiras em função da elevação da disponibilidade de P no solo também foram observados por FloresAylas et al. (2003). Para a espécie pioneira sabiá, contudo, a resposta não foi tão expressiva (Figura 4A).

R. Árvore, Viçosa-MG, v.32, n.5, p.799-807, 2008 

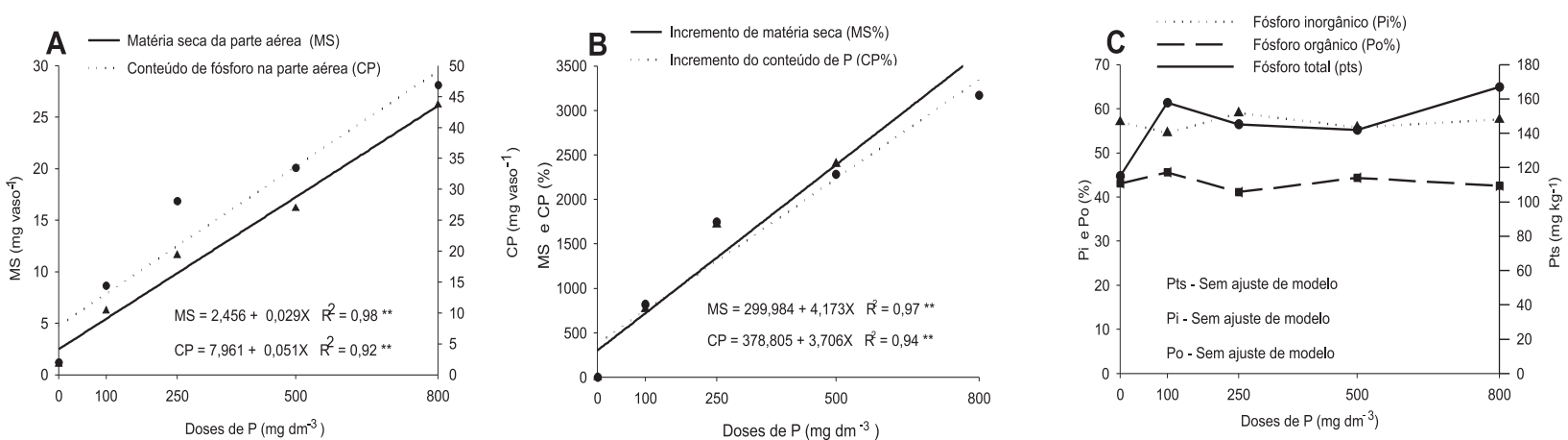

Figura 1 - Matéria seca (MS) e conteúdo de P(CP) na parte aérea (A), incrementos relativos de matéria seca (MS\%) e de conteúdo de $\mathrm{P}(\mathrm{CP} \%)(\mathrm{B})$ e distribuição das frações Pts, Pi e Po (C) da aroeira (Lithraea molleoides) em resposta a doses de fósforo.

Figure 1 - Shoot dry matter $(M S)$ and shoot $P$ content $(C P)(A)$, relative increments of dry matter $(M S \%)$ and $P$ content $(C P \%)$ $(B)$, and distribution of the P fractions (Pts, Pi and Po) (C) of Lithraea molleoides in response to phosphorus doses.
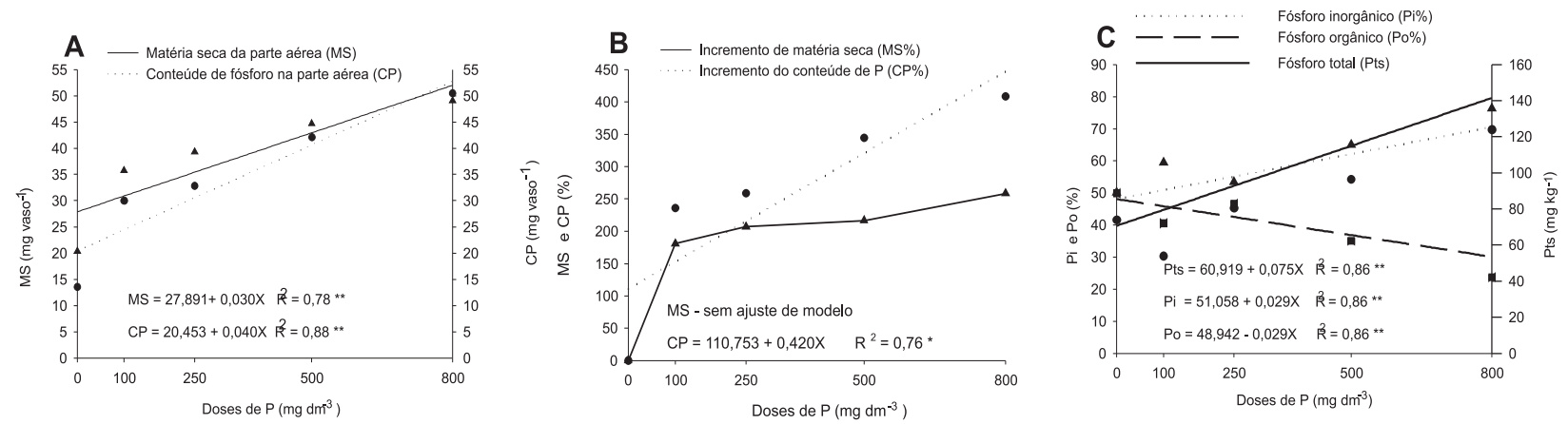

Figura 2 - Matéria seca (MS) e conteúdo de $\mathrm{P}(\mathrm{CP})$ na parte aérea (A), incrementos relativos de matéria seca (MS\%) e de conteúdo de $\mathrm{P}(\mathrm{CP} \%)$ (B) e distribuição das frações (Pts, Pi e Po) (C) da aroeirinha (Shcinus terebinthifolius) em resposta a doses de fósforo.

Figure 2-Shoot dry matter $(M S)$ and shoot $P$ content $(C P)(A)$, relative increments of dry matter $(M S \%)$ and $P$ content $(C P \%)(B)$, and distribution of the P fractions (Pts, Pi and Po) $(C)$ of Shcinus terebinthifolius in response to phosphorus doses.
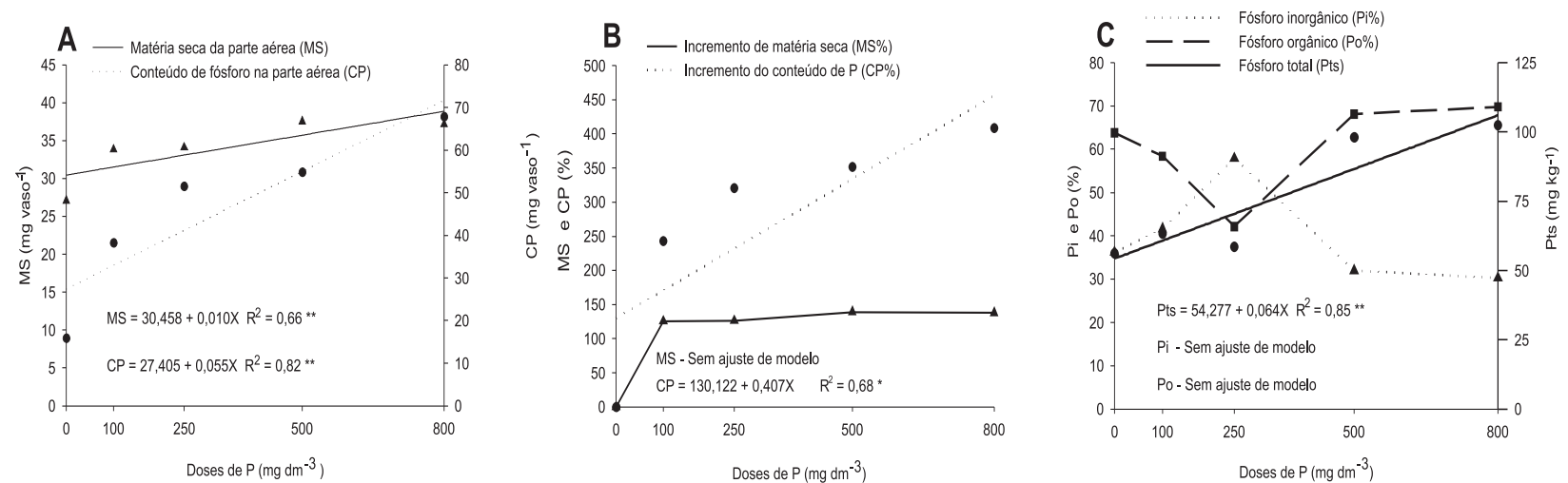

Figura 3 - Matéria seca (MS) e conteúdo de $\mathrm{P}(\mathrm{CP})$ na parte aérea (A), incrementos relativos de matéria seca (MS\%) e de conteúdo de $\mathrm{P}(\mathrm{CP} \%)$ (B), e distribuição das frações (Pts, Pi e Po) (C) da sesbânia (Sesbania virgata) em resposta a doses de fósforo.

Figure 3 - Shoot dry matter $(M S)$ and shoot $P$ content $(C P)(A)$, relative increments of dry matter $(M S \%)$ and $P$ content $(C P \%)$ $(B)$, and distribution of the P fractions (Pts, Pi and Po) (C) of Sesbania virgata in response to phosphorus doses.

R. Árvore, Viçosa-MG, v.32, n.5, p.799-807, 2008 

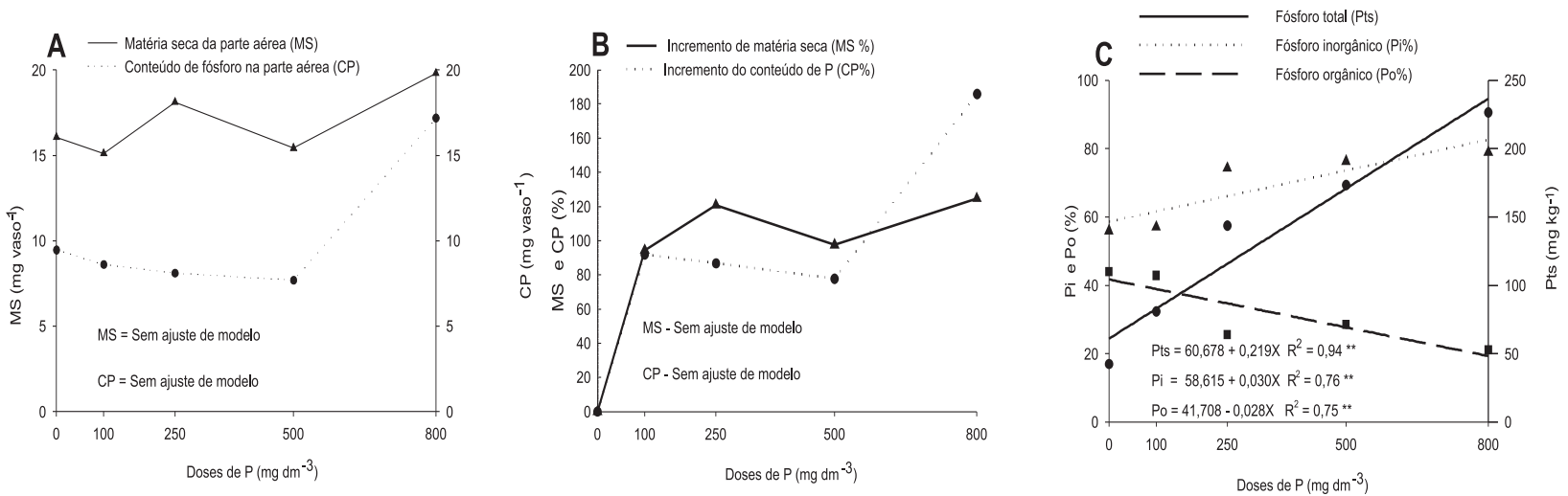

Figura 4 - Matéria seca (MS) e conteúdo de P (CP) na parte aérea (A), incrementos relativos de matéria seca (MS\%) e de conteúdo de P (CP\%) (B) e distribuição das frações (Pts, Pi e Po) (C)da sabiá (Mimosa caesalpiniaefolia) em resposta a doses de fósforo.

Figure 4-Shoot dry matter $(M S)$ and shoot $P$ content $(C P)(A)$, relative increments of dry matter $(M S \%)$ and $P$ content $(C P \%)(B)$, and distribution of the P fractions (Pts, Pi and Po) (C) of Mimosa caesalpiniaefolia in response to phosphorus doses.

No caso das espécies clímácicas guanandi e óleobálsamo, praticamente não houve alteração do crescimento em função do aumento da dose de P (Figuras 5A e 6A). Isso indica menor exigência do nutriente por essas espécies, quando comparadas com as demais. Avaliando o comportamento das mudas de espécies clímácicas em campo, Lima et al. (1997) observaram que elas não apresentaram resposta ao fornecimento de $\mathrm{P}$ aos oito meses pós-plantio. Essa diferença de comportamento das espécies clímácicas em relação às pioneiras se deve, em parte, ao fato de as pioneiras apresentarem sistema radicular mais desenvolvido e maior densidade de raízes finas, o que propicia taxas de crescimento e de absorção de nutrientes mais elevadas (GONÇALVES et al., 1992ab). Também, outros fatores como maiores tamanho e peso de sementes permitiriam maior conteúdo de $\mathrm{P}$ na forma de compostos de reserva que atenderiam à demanda pelo nutriente numa etapa inicial do crescimento dessas espécies climácicas (FURTINI et al., 2000). Aliado a isso, uma baixa eficiência de utilização de $\mathrm{P}$ apresentado por espécies de crescimento lento poderia contribuir para a pequena resposta ao fornecimento do nutriente (LAMBERS e POORTER, 1992).

O padrão de resposta das espécies quanto ao acúmulo de P na parte aérea (CP) também foi variável. À exceção da espécie pioneira sabiá (Figura 4A) e das climácicas guanandi e óleo-bálsamo (Figuras 5A e 6A), as demais apresentaram gradativo acúmulo de $\mathrm{P}$ em resposta ao aumento das doses fornecidas (Figuras 1A, 2A, 3A e 7A). Esses resultados confirmam, como tendência geral, a maior capacidade de absorção de $\mathrm{P}$ das espécies pioneiras. Esse comportamento diferencial permite inferir que as diferenças genéticas e fisiológicas entre os grupos sucessionais (pioneiras x clímácicas), que controlam também a morfologia do sistema radicular, são os principais reguladores da taxa de absorção e do conteúdo de P na parte aérea (GONÇALVES et al., 1992ab).

As respostas avaliadas em termos de incrementos relativos na produção de matéria seca da parte aérea (MS\%) e no conteúdo de P na parte aérea (CP\%) permitem discriminar melhor os padrões de comportamento das espécies estudadas. Entre as espécies pioneiras, a aroeira apresenta estreita correspondência $(r=0,98 * *)$ entre os incrementos de matéria seca e de conteúdo de P em função da adubação (Figura 1B). Já as espécies aroeirinha e sesbânia caracterizaram-se por apresentar incrementos lineares no conteúdo de $\mathrm{P}$, porém não acompanhados de aumento proporcional do crescimento (Figuras 2B e 3B). Esses resultados indicam ter havido baixa conversão do $\mathrm{P}$ absorvido em biomassa, o que implica menor eficiência de utilização do nutriente por essas espécies ou consumo de luxo nessa fase de crescimento, em que grande parte do $\mathrm{P}$ não foi utilizado para produção de matéria seca. No caso da espécie pioneira sabiá e das clímácicas guanandi e óleo-bálsamo, os incrementos, tanto na produção de matéria seca quanto no acúmulo de $\mathrm{P}$, foram pouco consistentes em relação ao aumento na disponibilidade de $\mathrm{P}$ (Figuras $4 B, 5 B$ e $6 B)$.

R. Árvore, Viçosa-MG, v.32, n.5, p.799-807, 2008 

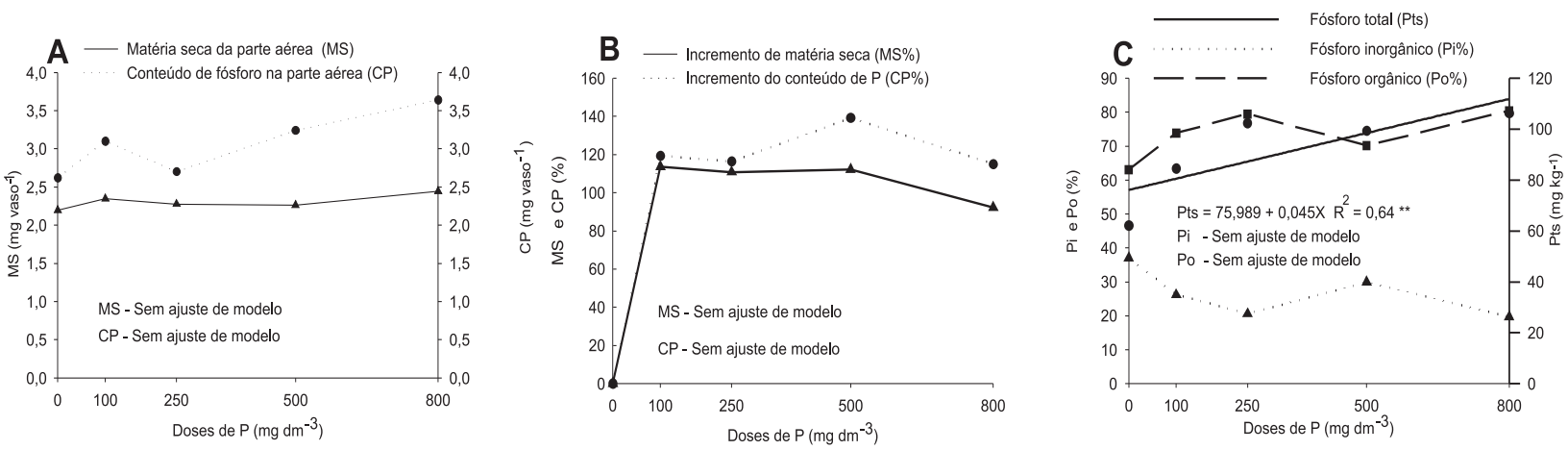

Figura 5 - Matéria seca (MS) e conteúdo de P (CP) na parte aérea (A), incrementos relativos de matéria seca (MS\%) e de conteúdo de $\mathrm{P}(\mathrm{CP} \%)$ (B) e distribuição das frações (Pts, Pi e Po) (C) (C) do guanandi (Calophyllum brasiliensis) em resposta a doses de fósforo.

Figure 5 - Shoot dry matter $(M S)$ and shoot $P$ content $(C P)(A)$, relative increments of dry matter $(M S \%)$ and $P$ content $(C P \%)(B)$, and distribution of the P fractions (Pts, Pi and Po) (C) of Calophyllum brasiliensis in response to phosphorus doses.
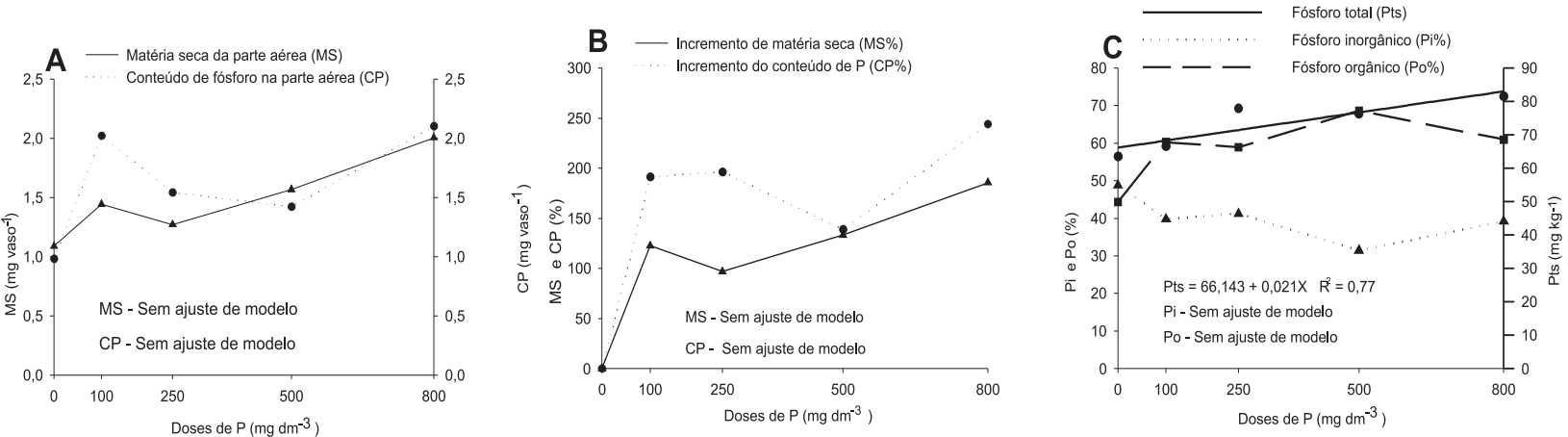

Figura 6 - Matéria seca (MS) e conteúdo de P na parte aérea (CP) (A), incrementos relativos de matéria seca (MS\%) e de conteúdo de $\mathrm{P}(\mathrm{CP} \%)$ (B) e distribuição das frações de P (Pts, Pi e Po) (C) do óleo- balsamo (Myroxylon peruiferum) em resposta a doses de fósforo.

Figure 6-Shoot dry matter $(M S)$ and shoot $P$ content $(C P)(A)$, relative increments of dry matter $(M S \%)$ and $P$ content $(C P \%)(B)$, and distribution of the P fractions (Pts, Pi and Po) (C) of Myroxylon peruiferum in response to phosphorus doses.
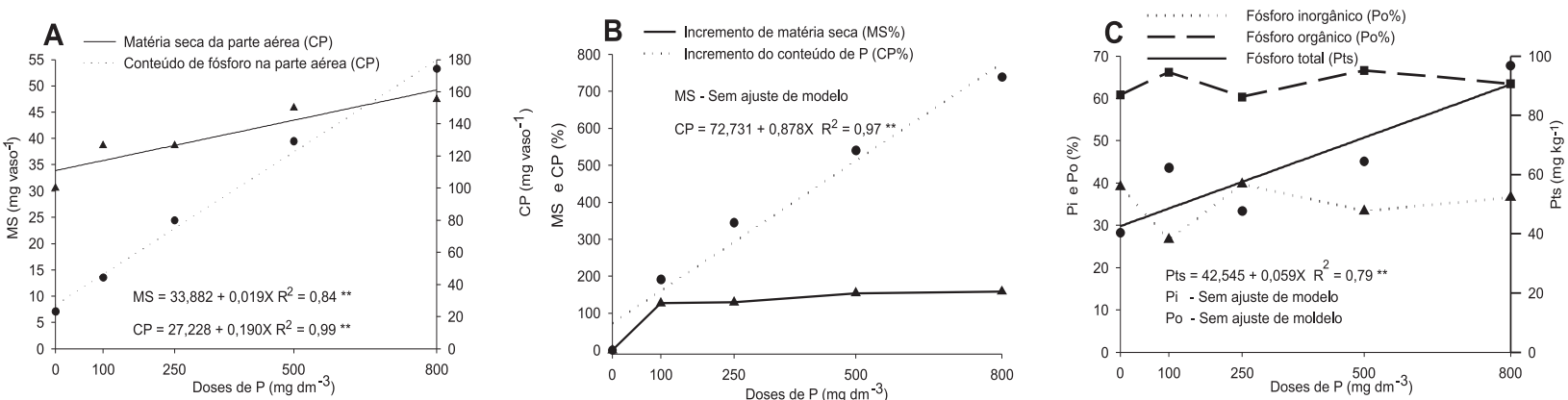

Figura 7 - Matéria seca (MS) e conteúdo de P (CP) na parte aérea (A), incrementos relativos de matéria seca (MS\%) e de conteúdo de $\mathrm{P}(\mathrm{CP} \%)$ (B) e distribuição das frações (Pts, Pi e Po) (C) do jatobá (Hymenaea courbaril) em resposta a doses de fósforo.

Figure 7 - Shoot dry matter $(M S)$ and shoot $P$ content $(C P)(A)$, relative increments of dry matter $(M S \%)$ and $P$ content $(C P \%)(B)$, and distribution of the P fractions (Pts, Pi and Po) (C) of Hymenaea courbaril in response to phosphorus doses.

R. Árvore, Viçosa-MG, v.32, n.5, p.799-807, 2008 
A existência de comportamentos contrastantes, principalmente entre os grupos de pioneiras e clímácicas, possivelmente se deve aos diferentes ajustes metabólicos proporcionados pelas diferenças genéticas entre as espécies, que podem afetar a taxa de crescimento ou absorção de $\mathrm{P}$ e sua retranslocação no interior da planta e, conseqüentemente, influenciando a produção de biomassa pelas espécies (FURTINI NETO et al., 1998; PAULA et al., 2003; RESENDE et al., 2005). Essas diferenças nos ajustes metabólicos podem resultar em vantagem ou desvantagem adaptativa às condições de fertilidade do solo (BIELESKI e FERGUSON, 1983).

A eficiência de utilização de $\mathrm{P}$ por dada espécie reflete o seu potencial em converter o $\mathrm{P}$ disponível no solo em biomassa (ANGHINONI, 2003). Resultados similares aos observados em aroeirinha, sesbânia e jatobá (Figuras 2B, 3B e 7B) foram obtidos por Paula et al. (2003), avaliando o controle genético na eficiência de utilização de fósforo em famílias de meios-irmãos de Eucalyptus grandis. Esses autores verificaram baixa produção de matéria seca na presença de elevados teores de $\mathrm{P}$ na folha e no caule com aumento da dose de 200 para $400 \mathrm{mg} \mathrm{dm}^{-3} \mathrm{de}_{2} \mathrm{O}_{5}$. Geralmente, quando uma planta se encontra numa situação de baixa disponibilidade de $\mathrm{P}$, ela tende a utilizá-lo de forma mais eficiente, contrariamente ao que se observa quando é submetida a uma condição de maior fertilidade (BALIEIRO et al., 2001). Esse padrão parece não se aplicar às espécies clímácicas deste estudo, os quais apresentaram crescimento lento e relativa indiferença à disponibilidade de P no solo. Segundo Furtini Neto et al. (2000) e Resende et al. (2005), esse comportamento está associado ao baixo crescimento inicial das espécies climácicas e maiores reservas de $\mathrm{P}$ nas sementes, estando este último fator ligado diretamente ao tamanho e peso da semente.

\subsection{Frações inorgânica e orgânica de fósforo na folha}

De modo geral, as espécies apresentaram aumento no teor de Pts à medida que se elevaram as doses de $\mathrm{P}$, notadamente nas doses mais elevadas do nutriente (Figuras 2C a 7C), exceção feita à aroeira (Figura 1C). Em relação à aroeira, esse comportamento pode ser em função do efeito-diluição do nutriente nos tecidos da planta, consequiência do rápido crescimento da espécie.

No tocante às frações fosfatadas, observou-se que as espécies aroeira, guanandi, óleo-bálsamo e jatobá (Figuras 1C, 5C, 6C e 7C) apresentaram menores alterações na dinâmica do Pi e Po em função do aumento das doses de $\mathrm{P}$, indicando que o nutriente adicionalmente absorvido foi preferencialmente convertido para formas orgânicas, em atendimento à demanda nutricional, mantendo sempre a mesma proporção de Pi e Po.

Aroeira, aroeirinha e sabiá acumularam fósforo preferencialmente na forma de Pi (Figuras 1C, 2C e 4C), com incrementos proporcionalmente maiores de $\mathrm{P}$ nesse compartimento, conforme o aumento nas doses do nutriente. Em aroeirinha, esse comportamento já havia sido relatado por Fernandes et al. (2000a). Essa forma de partição do $\mathrm{P}$ absorvido reforça a idéia de que essas espécies apresentam eficiência de conversão mais limitada. Nesse caso, a maior parte do P ficaria armazenada no vacúolo na forma de Pi (BIELESKI e FERGUNSON, 1983), e, uma vez bem supridas, essas espécies suportariam por mais tempo interrupção no fornecimento do nutriente antes de paralisar o crescimento, pois ocorreria mobilização das reservas de Pi vacuolar para o citoplasma, garantindo o metabolismo normal das plantas(FURTININETOetal., 1996; FERNANDES et al., 2000b; MARTINEZ et al., 2005).

Sesbânia, guanandi, óleo-bálsamo e jatobá apresentaram predomínio de $\mathrm{P}$ na forma orgânica (Figuras 3C, 5C, 6C e 7C). Em relação as espécies guanandi e óleo-bálsamo, esse comportamento seria justificado em função da reduzida capacidade de absorção de $\mathrm{P}$ (Figuras 5A e 6A), pois o crescimento lento implicaria menor desenvolvimento do sistema radicular e exploração do solo e, conseqüentemente, menor absorção de P. Assim, maior proporção do $\mathrm{P}$ absorvido é convertido em compostos orgânico no citoplasma. Maior participação de Po foi observada por Fernandes et al. (2000a) em folhas de paineira (espécie clímácica exigente de luz) quando comparada com aroeirinha (pioneira) e jambolão (clímácica tolerante à sombra). Segundo Martinez et al. (2005), a fração orgânica é a que sofre a menor variação em função de alteração no fornecimento de P nas plantas. Em termos práticos, tais observações permitem inferir que as espécies sesbânia, guanandi, óleo-bálsamo e jatobá podem ser utilizadas para reflorestar áreas de solos com baixos teores de $\mathrm{P}$, pois o seu metabolismo e crescimento inicial são pouco influenciados pela disponibilidade do nutriente.

Fernandes et al. (2000a) sugeriram que, se o ritmo de crescimento da planta for lento ou a demanda de $\mathrm{P}$ for pequena, maior fornecimento do nutriente pode resultar no acúmulo de Pi. No entanto, tal observação foi confirmada apenas para algumas espécies pioneiras neste estudo. No caso das espécies climácicas, parece haver rígido ajuste entre a atividade metabólica e a absorção de $\mathrm{P}$, de forma que o lento crescimento limita

R. Árvore, Viçosa-MG, v.32, n.5, p.799-807, 2008 
a absorção do nutriente presente no solo. Além de não necessitar de elevadas doses do nutriente para suportar o crescimento inicial, a utilização de fontes de menor solubilidade seria uma prática de manejo relativamente segura, pois liberação mais lenta do $\mathrm{P}$ do fertilizante garantiria o crescimento satisfatório da planta por considerável período de tempo.

Maior participação das formas de Po após o período de omissão de $\mathrm{P}$ foi observado por Furtini Neto et al. (1998), avaliando frações fosfatadas em mudas de Eucalyptus. Isso indica que essas espécies mobilizam sua reserva de Pi para manter o crescimento. No caso das espécies clímácicas deste estudo, evidencia-se forte restrição na demanda nutricional por P, visto que a absorção é pequena e há menor participação do Pi mesmo em condições de maior disponibilidade do nutriente. Desse modo, a planta não se desenvolve muito e nem dispõe de reservas de $\mathrm{P}$ para crescimento posterior, o qual seria, então, mais dependente de uma eficiente ciclagem interna do nutriente (remobilização).

\section{CONCLUSÕES}

1. As espécies florestais estudadas apresentam grande variação no comportamento diante da adubação fosfatada. As espécies pioneiras foram mais eficientes em produzir matéria seca da parte aérea e na absorção de P que as climácicas.

2. Entre as espécies pioneiras, a aroeira teve produção de biomassa estreitamente relacionada ao acúmulo de $\mathrm{P}$, de modo que as proporções de Pi e Po permaneceram inalteradas com o aumento da disponibilidade de P. O crescimento de aroeirinha, sesbânia e jatobá não acompanhou a absorção do nutriente, que é então acumulado na forma de Pi na primeira e Po nas últimas.

3. As espécies clímácicas guanandi e óleo-bálsamo apresentaram maior presença de Po, porém a absorção do nutriente e o crescimento das plantas foram pouco afetados pela adubação fosfatada.

\section{REFERÊNCIAS}

ANGHINONI, I. Fatores que interferem na eficiência da adubação fosfatada. In: SIMPÓSIO SOBRE FÓSFORO NAAGRICULTURABRASILEIRA, 2003, Piracicaba. Anais... Piracicaba: Potafos/Anda, 2003. CD-ROM.

BALIEIRO, F. C.; OLIVEIRA, I. G.; DIAS, L.E. Formação de mudas de Acacia holosericea e Acacia auriculiformis: resposta a calagem, fósforo, potássio e enxofre. Revista Árvore, v.25, n.2, p.183-191, 2001.

R. Árvore, Viçosa-MG, v.32, n.5, p.799-807, 2008
BIELESKI, R. L.; FERGUSON, J. B. Physiology and metabolism of phosphate and its compounds. In: LACHLI, A.; CIELESKI, R. L. (Eds.) Encyclopedia of plant physiology. Inorganic plant nutrition. Berlin: Spring, 1983. p.422-449. (New series, v.15A).

DAVIDE, A. C.; FARIA, J. M. R.; BOTELHO, S. A. Propagação de espécies florestais. Lavras: Universidade Federal de Lavras, 1995. 41p.

EMPRESA BRASILEIRA DE PESQUISA AGROPECUÁRIA - EMBRAPA. Centro Nacional de Pesquisa de Solos. Sistema brasileiro de classificação de solos. Rio de Janeiro: 1999. 412p.

FABRES, A. S. et al. Níveis críticos de diferentes frações de $\mathrm{P}$ em plantas de alface cultivadas em diferentes solos. Revista Brasileira de Ciência do Solo, v.11, n.1, p.51-57, 1987.

FERNANDES, L. A. et al. Frações de fósforo e atividade da fosfatase ácida em plantas de feijoeiro cultivadas em solos de várzea. Revista Brasileira de Ciência do Solo, v.24, n.3, p.561-571, 2000b.

FERNANDES, L. A. et al. Crescimento inicial, níveis críticos de fósforo e frações fosfatadas em espécies florestais. Pesquisa Agropecuária Brasileira, v.35, n.6, p.1191-1198, 2000a.

FERREIRA, D. F. Análises estatísticas por meio do Sisvar para Windows versão 4.0. In: REUNIÃO ANUAL DA REGIÃO BRASILEIRA DA SOCIEDADE INTERNACIONAL DE BIOMETRIA, 45., 2000, São Carlos. Programa e

resumos... São Carlos: Universidade Federal de São Carlos, 2000. p.255-258.

FLORES-AYLAS, W. W. et al. Efeito de Glomus etunicatum e fósforo no crescimento inicial de espécies arbóreas em semeadura direta.

Pesquisa Agropecuária Brasileira, v.38, n.2, p. 257-266, 2003.

FREIRE, J. C. et al. Resposta do milho cultivado em casa de vegetação à níveis de água em solos da região de Lavras-MG. Revista Brasileira de Ciência do Solo, v.4, n.5, p.5-8, 1980. 
FURTINI NETO, A. E. Eficiência nutricional, cinética de absorção e frações

fosfatadas em Eucalyptus spp. 1994. 99f. Tese

(Doutorado em Solos e Nutrição de Plantas) -

Universidade Federal de Viçosa, Viçosa, MG, 1994.

FURTINI NETO, A. E. et al. Eficiência nutricional de mudas de Eucalyptus em relação a fósforo.

Revista Árvore, v.20, n.1, p.17-28, 1996.

FURTINI NETO, A. E. et al. Fertilização em reflorestamento com espécies nativas. In: GONÇALVES, J. L. M. \& BENEDETTI, V.

Nutrição e fertilização florestal. Piracicaba:IPEF, 2000. p.352-379.

FURTINI NETO, A. E. et al. Frações fosfatadas em mudas de eucaliptus. Revista Brasileira de Ciência do Solo, v.22, n.2, p.267-274, 1998.

GONÇALVES, J. L. M. et al. Produção de biomassa e sistema radicular de espécies de diferentes estágios sucessionais. Revista do Instituto Florestal, v.4, p 363-367, 1992b.

GONÇALVES, J. L. M. et al. Capacidade de absorção e eficiência nutricional de algumas espécies arbóreas tropicais. Revista do Instituto Florestal, v.4, p. 463-469, 1992a.

HOGUE, E.;WILCOX, G. E.; CANTLIFFE, D. J. Effect soil phosphorus levels on phosphate fractions in tomato leaves. American Society for Horticultural Science Journal, v.95, n.1, p.174-176, 1970.

LAMBERS, H.; POORTER, H. Inherent variations in growth rate between higher plants: A search for fisiological causes and ecological consequences. Advances in Ecological Research, v.23, p.188-261, 1992.

LIMA, H. N. et al. Crescimento inicial a campo de sete espécies arbóreas nativas em resposta à adubação mineral com NPK.Ciência e Agrotecnologia, v.21, n.1, p.189-195, 1997.

MALAVOLTA, E.; VITTI, G. C.; OLIVEIRA, S. A. Avaliação do estado nutricional das plantas: princípios e aplicações. 2.ed.

Piracicaba: POTAFOS, 1997. 319p.
MARSCHNER, H. Mechanisms of adpatation of plants to acid soils. Plant and Soil, v.134, n.1, p.1-20, 1991.

MARTineZ, H. E. P. Cinética da absorção e das frações fosfatadas em sete variedades de soja (Glicine Max L. (Merril)). Viçosa, MG: Universidade Federal de Viçosa, 1992. Não Paginada. (Relatório final de Pós-Doutoramento)

MARTINEZ, H. E. P. et al. Phosphate forms in plant and their internal buffering in five soybean cultivars. Revista Brasileira de Ciência do Solo, v.29, p.249-257, 2005.

PAULA, R. C. et al. Controle genético da eficiência de utilização de fósforo em famílias de meios-irmãos de Eucalyptus grandis, em casa de vegetação.

Revista Ârvore, v.27, n.1, p.25-34, 2003.

RESENDE, A. V. Nutrição e crescimento de espécies florestais de diferentes grupos sucessionais em resposta à fertilização fosfatada na fase de mudas. 1997. 81f. Dissertação (Mestrado em Solos e Nutrição de Plantas) - Universidade Federal de Lavras, Lavras, 1997.

RESENDE, A. V.; FURTINI NETO, A. E.; CURI, N. Mineral nutrition and fertilization of native tree species in Brazil: research progress and suggestions for management. Journal of Sustainable Forestry, v.20, n.2, p.45-81, 2005.

SCHUMACHER, M. V.; CECONI, D. E.; SANTANA, C. A. Influência de diferentes doses de fósforo no crescimento de mudas de angico-vermelho (Parapiptadenia rigida (Bentham) Brenan). Revista Árvore, v.28, n.1, p.149-155, 2004.

SIQUEIRA, J. O. et al. Aspectos de solos, nutrição vegetal e microbiologia na implantação de matas ciliares. Belo Horizonte: CEMIG, 1995. 28p.

SMILLE, R. M.; KROTKOV, G. The estimation of nucleic acids in some algae and higher plants. Canadian Journal of Botany, v.38, n.1, p.31-49, 1960.

R. Árvore, Viçosa-MG, v.32, n.5, p.799-807, 2008 
